\title{
Co-simulation of Six DOF Wire Driven Parallel Mechanism Based on ADAMS and Matlab
}

\author{
Aofei Tang ${ }^{1, a}$, Shujuan $\mathrm{Li}^{1}$, Yunfei $\mathrm{Wu}^{1}$, Xiaohua Fang ${ }^{1}$ \\ ${ }^{1}$ School of Mechanical and Precision Instrumental Engineering, Xi'an University of Technology, Xi'an 710048, P. R. China
}

\begin{abstract}
The dynamic model of the 6 DOF Wire Driven Parallel Mechanism (WDPM) system is introduced. Based on MATLAB system, the simulation of the inverse dynamic model is achieved. According to the simulation result, the mechanical model for the WDPM system is reasonable. Using ADAMS system, the dynamic model of the virtual prototype is verified by the simulation analysis. The combined control model based on ADAMS/Simulink is derived. The WDPM control system is designed with MATLAB/Simulink. The torque control method is selected for the outer ring and the PD control method for the inner ring. Combined with the ADAMS control model and control law design, the interactive simulation analysis of the WDPM system is completed. According to the simulation results of the spatial circle tracking and line tracking at the end of the moving platform, the tracking error can be reduced by the designed control algorithm. The minimum tracking error is $0.2 \mathrm{~mm}$ to $0.3 \mathrm{~mm}$. Therefore, the theoretical foundation for designing hardware systems of the WDPM control system is established.
\end{abstract}

\section{Introduction}

WDPM utilizes flexible wire instead of connecting rod as the traction element which make it quite different from the normal parallel mechanism. As the flexible wire can only bear unidirectional loads, the performance of Wire Driven Parallel Mechanism (WDPM) is important for actual application. More and more research works have been done by the researchers of many countries [1-4]. As a result, WDPM is widely used in engineering fields recently, such as machining, tracking of the large spherical radio telescope, wind tunnel testing of airplane etc. Therefore, research on the WDPM has significant value on academic research and engineering application.

\section{Dynamics analysis}

With the rapid development of mechanical system, the need to achieve a lightweight mechanical system has become an important factor with respect to design efficiency issues and saving energy. It also made the system flexibility to be a considerable problem. Compared with traditional rigid arm, flexible arm has unique physical properties that cause the research work more complex. To solve the vibration problem is the key issue for flexible system. So the research method for studying flexible system is quite import, the assumed mode method and finite element method are often used for theoretical model research [5-7]. Comprehensive studying of simulation and experiment can be helpful to the optimization of flexible system, and is beneficial to the simulation research of the control method.

\footnotetext{
a Corresponding author: aofeitang@126.com
}

\subsection{Dynamic model of WDPM}

Linear system can be modeled discretely in ADAMS, the deformation of each node, which is composed of a plurality of linear modal superposition, is considered normally. There are three methods of flexible wire modeling:

(1) Create flexible body directly to generate a flexible wire model.

(2) Create some rigid cylinder bodies and connected with bushings to simulate flexible wire.

(3) Select rotation joints connecting some cylinder bodies to simulate flexible wire.

The second method is selected for modeling, ten cylinders are connected by bushings, six components of the force and torque are defined as [Fx Fy Fz Tx Ty Tz], the force model is shown in Fig. 1.

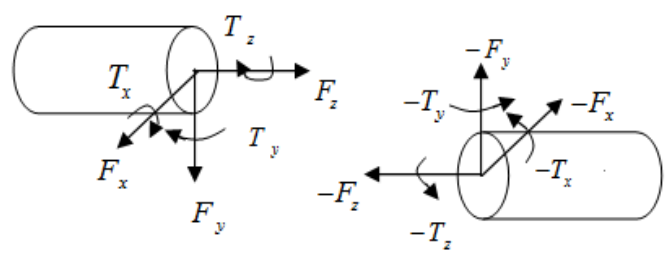

Fig. 1 Cylinder force modeling

Coordinate marking the cylinder center, assign I and $\mathrm{J}$ respectively, and assign $K$ as stiffness coefficient, $C$ as damping coefficient, $x_{0}, y_{0}$ and $z_{0}$ as the initial values of 
each coordinate axis, $x, y$ and $z$ as the directions of each coordinate axis.

$$
\left[\begin{array}{l}
F_{x} \\
F_{y} \\
F_{z} \\
T_{x} \\
T_{y} \\
T_{z}
\end{array}\right]=-\left[\begin{array}{cccccc}
K_{11} & 0 & 0 & 0 & 0 & 0 \\
0 & K_{22} & 0 & 0 & 0 & 0 \\
0 & 0 & K_{33} & 0 & 0 & 0 \\
0 & 0 & 0 & K_{44} & 0 & 0 \\
0 & 0 & 0 & 0 & K_{55} & 0 \\
0 & 0 & 0 & 0 & 0 & K_{66}
\end{array}\right]\left[\begin{array}{c}
x \\
y \\
z \\
a \\
b \\
c
\end{array}\right]
$$

$$
-\left[\begin{array}{cccccc}
C_{11} & 0 & 0 & 0 & 0 & 0 \\
0 & C_{22} & 0 & 0 & 0 & 0 \\
0 & 0 & C_{33} & 0 & 0 & 0 \\
0 & 0 & 0 & C_{44} & 0 & 0 \\
0 & 0 & 0 & 0 & C_{55} & 0 \\
0 & 0 & 0 & 0 & 0 & C_{66}
\end{array}\right]\left[\begin{array}{c}
V_{x} \\
V_{y} \\
V_{z} \\
\omega_{x} \\
\omega_{y} \\
\omega_{z}
\end{array}\right]+\left[\begin{array}{c}
F_{1} \\
F_{2} \\
F_{3} \\
T_{1} \\
T_{2} \\
T_{3}
\end{array}\right]
$$

Where $\mathrm{F}$ and $\mathrm{T}$ denote force and torque, $\mathrm{x}, \mathrm{y}, \mathrm{z}, \mathrm{a}, \mathrm{b}, \mathrm{c}, \mathrm{vx}$, $\mathrm{vy}, \mathrm{vz}, \omega \mathrm{x}, \omega \mathrm{y}, \omega \mathrm{z}$ denote the relative displacement, rotation angle, velocity, angular velocity between I MARKER and J MARKER respectively. Kii and Cii denote the stiffness coefficient and damping coefficient of the bushing. F1, F2, F3, T1, T2, T3 denote the initial values of the force and torque along three coordinate axis. Create a dynamic model of WDPM system in ADAMS/View, the parameters of bushing that composing the flexible wire are shown in Table 1.

Table 1. Basic parameters of bushing

\begin{tabular}{rcccc}
\hline Parameter & Stiffness & Damp & $\begin{array}{c}\text { Torsional } \\
\text { rigidity }\end{array}$ & $\begin{array}{c}\text { Torsional } \\
\text { damping }\end{array}$ \\
\hline Value & 59346 & 1 & 0.1 & 1 \\
\hline
\end{tabular}

The moving platform is dragged by seven flexible wires, each flexible wire is connected with the moving platform by spherical joint, and is connected with the framework with hooke joint. The creation direction of the spherical joint and hooke joint $\mathrm{s}$ along the flexible wire. In order to allow the flexible wire to move back and forth freely, use one translational joint in the flexible wire. The dynamic model of WDPM is shown in Fig. 2.

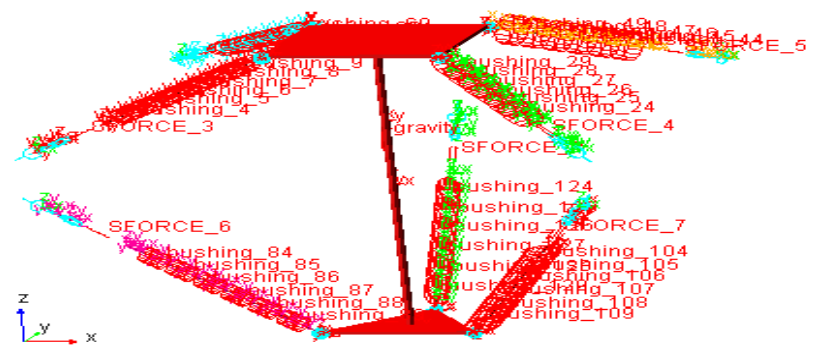

Fig. 2 Dynamic model of WDPM

The ADAMS model of the 6-DOF WDPM is created according to the mechanism parameters which is listed in Table 2.
Table 2 Main parameters of 6-DOF WDPM mechanism

\begin{tabular}{c|c}
\hline Degree of freedom & 6 \\
\hline Numbers of Flexible wires & 7 \\
\hline DOF of spherical joint of moving platform & 3 \\
\hline DOF of Hook joint of static platform & 2 \\
\hline Numbers of translational joint & 7 \\
\hline Triangle side length of moving platform $(\mathrm{mm})$ & 350 \\
\hline Rectangle dimension of moving platform $(\mathrm{mm})$ & 280 \\
\hline Mass of moving platform & $10 \mathrm{~kg}$ \\
\hline
\end{tabular}

\subsection{Exporting dynamic model of WDPM}

To export the dynamic model of WDPM, three stages maybe concerned.

(1) Create input state variables and connect with physical quantity of the model

After the dynamic simulation of the WDPM model is completed, the input parameters of the model need to connect with the state variables. The exported dynamic model proceed with data transfer by state variables when co-simulation is carried on. The forces in the model can be named as Force_i $(1,2, \ldots 7)$ which is defined as input state variables. Force is a variable of time, the force input to the model is calculated by external software and proceed with data transfer. The initial condition is $\mathrm{F}($ time,$\ldots)=0$.

Professional data receiving function can be used in ADAMS software, the input data of control model transfer to virtual prototype by state variables while the state variables and physical quantities are connected. The driving force function is defined as VARVAL (. platform. Force_i) which can transfer data to the input parameter variables of the virtual prototype as the external force is imported.

(2) Create output state variables

The input parameters are imported from external part and the initial values are parameters related to time, while the output variables are function expressions. The output variables of WDPM can be defined as

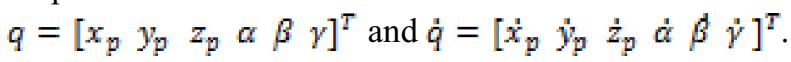
12 output state variables are created and named as Position_i(i=1,2..6) and Velocity_i $(\mathrm{i}=1,2,3,4,5,6)$.

(3) Export dynamic model

When the input and output state variables are defined in ADAMS/View, the dynamic model can be exported and three files are generated which are named as Controls_PD.m,Controls_PD.cmd and Controls_PD.adm [8-10].

\subsection{Create connecting module between MATLAB and ADAMS}

To export dynamic model of WDPM, ADAMS/Control module should be used. Select input and output parameters and generate files named Controls_PD.m to save input and output information, Controls_PD.cmd to 
transfer command, Controls_PD.adm to save data. Controls PD is the file name of export model. In MATLAB $\bar{B}$, input ADAMS_sys in command line to create the control system model as shown in Fig. 3

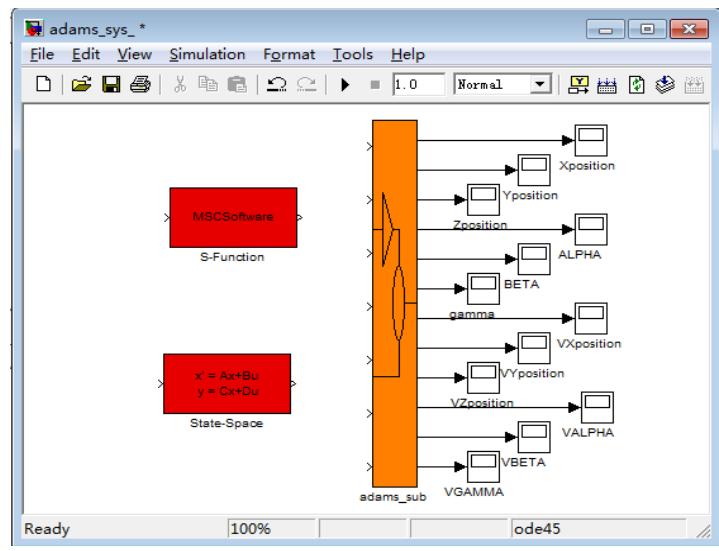

Fig. 3 Interface module of ADAMS and MATLAB

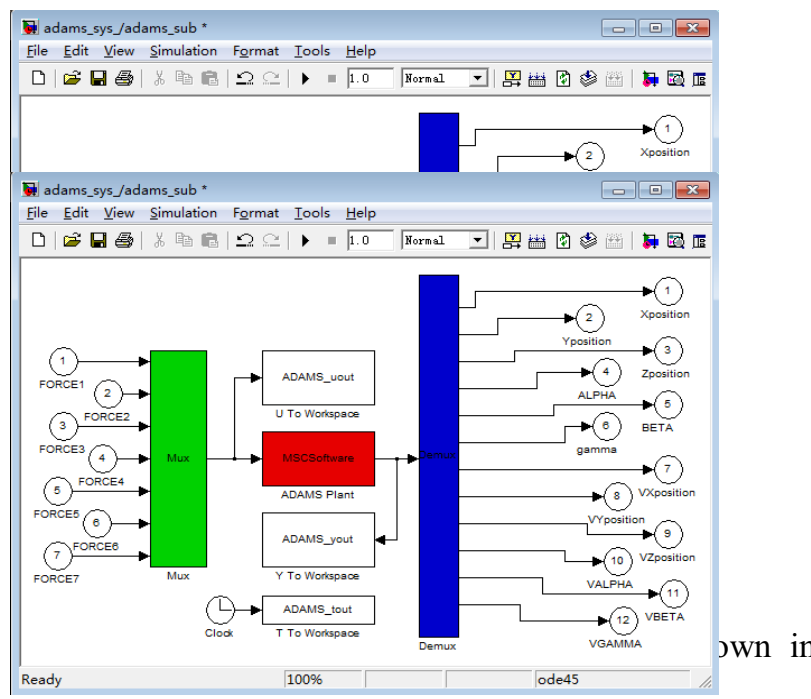

Fig. 4. In the module parameter dialog box, the animation mode is set to interactive in order to real-time observe the simulation process and intuitively study the motion of the moving platform.

\section{Create MATLAB control model}

\subsection{Dynamic characteristic of WDPM}

The dynamic model of 6-DOF WDPM can be defined as Equation (2) by Euler-Lagrangen.

$$
M \ddot{q}+N \dot{q}-W=J^{T} F
$$

Where $\mathrm{M}$ is Inertia matrix, $\mathrm{N}$ is Coriolis matrix, $\mathrm{W}$ is Gravity matrix, $\mathrm{J}$ is Jacobian matrix, $\dot{q}$ is Joint velocity matrix, $\ddot{q}$ is Joint acceleration vector, $\mathrm{F}$ is Joint force vector.

\subsection{Inner ring control system of WDPM}

Dynamic control is a kind of control mode based on computational torque method, according to the dynamic model of the controlled object, the control force or torque can be calculated by solving dynamic inverse solution. The control effect of the dynamic control system has a high dependence on the accuracy of the dynamic model of the controlled object. Therefore, It is only in the case of the complete accuracy of the dynamic model of the WDPM system, the precise control can be achieved.

If $q_{\mathrm{d}}$ denotes the expected tracking motion of WDPM platform, the error between the actual tracking and ideal tracking of the moving platform is defined as tracking error $e$.

$$
e=q_{d}-q
$$

Where $e=\left[e_{x} e_{y} e_{z} e_{\alpha} e_{\beta} e_{\gamma}\right]^{T}$, each component indicates the tracking error of the moving platform in the direction of $x, y, z, \alpha, \beta, \gamma$. If adopting fixed point control, $q_{\mathrm{d}}$ is a constant, so $\dot{q}_{d}=\ddot{q}_{d} \equiv 0$.

Make a derivation of Equation (3)

$$
\dot{e}=\dot{q}_{d}-\dot{q}, \ddot{e}=\ddot{q}_{d}-\ddot{q}
$$

Substituting the dynamic model into the Equation (4)

$$
\ddot{\theta}=\ddot{q}_{d}+M(q)^{-1}\left(N(q, \dot{q}) \dot{q}+W(q)-J^{T} F\right)
$$

Define the control input function as

$$
h=\ddot{q}_{d}+M(q)^{-1}\left(N(q, \dot{q}) \dot{q}+W(q)-J^{T} F\right)
$$

or

$$
M(q)\left(\ddot{q}_{d}-h\right)+N(q, \dot{q}) \dot{q}+W(q)=J^{T} F
$$

Equation (7) is control law of calculating torque.

\subsection{Design of control law for outer ring}

The position and attitude adjustment of moving platform, extremity actuator of WDPM, is achieved by the tension force changing of the flexible wire. To design the control law, the normal method is to proceed with trajectory planning, then solve the reverse solution by dynamic function, thus the tension component of each flexible wire is calculated respectively. Because WDPM system is quite complicated to control with the tension force as control object, select classical control theory to proceed with the control design of outer ring. As the PD control has outstanding control ability, assistant control signal $\mathrm{H}$ (t) is selected as PD feedback.

$$
D=-k_{d} \cdot \dot{e}-k_{p} \cdot e
$$

Where $k_{p}$ is proportion coefficient, $k_{d}$ is differential coefficient.

Thus the trajectory tracking control law of the WDPM platform based on reverse force compensation control is

$$
M(q)\left(\ddot{q}_{d}+k_{d} \dot{e}+k_{p} \theta\right)+N(q, \dot{q}) q+G(q)=J^{T} F
$$


The error function derived from Equation (9) is

$$
\ddot{\theta}+k_{d} \dot{\theta}+k_{p} e=0
$$

\subsection{Control model of MATLAB}

Create an interactive control system of the WDPM in MATLAB/Simulink as shown in Fig. 5.

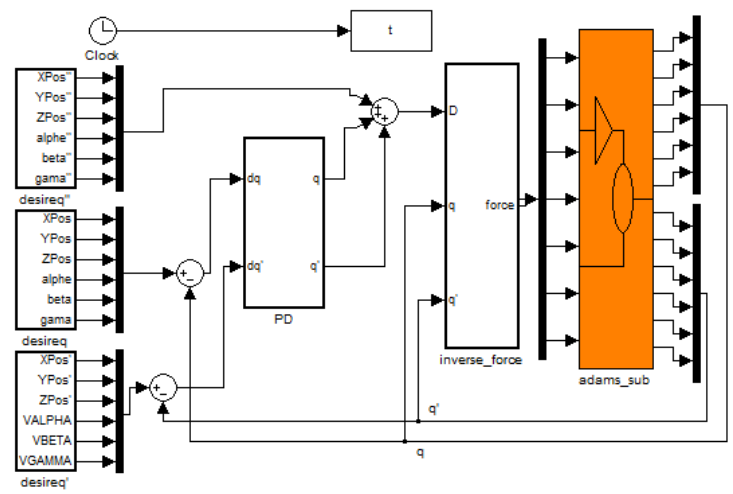

Fig. 5 Scheme of Control model in MATLAB/Simulink

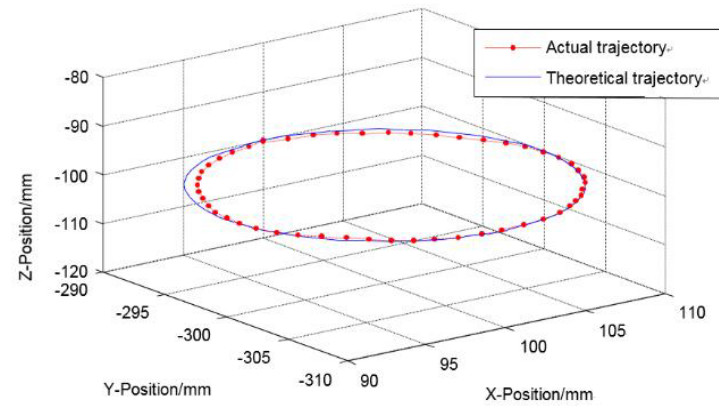

Fig. 6 Trajectory of the WDPM system

\section{Co-simulation experiment}

In the simulation, different simulation time can be set, control parameters $k_{p}=150, k_{d}=9$. Assume the moving platform is moving in a horizontal plane, the center point of the motion is $(100,-300,-100)$, radius is $10 \mathrm{~mm}$ and the start point is $(100,-290,-100)$. Let the moving platform make a translational motion in the simulation, simulation time is $10 \mathrm{~s}$. The trajectory Equation of the center point of the moving platform is

$$
\left\{\begin{array}{l}
x=100+10 \sin (t) \\
y=-300+10 \cos (t) \\
z=-100
\end{array}\right.
$$

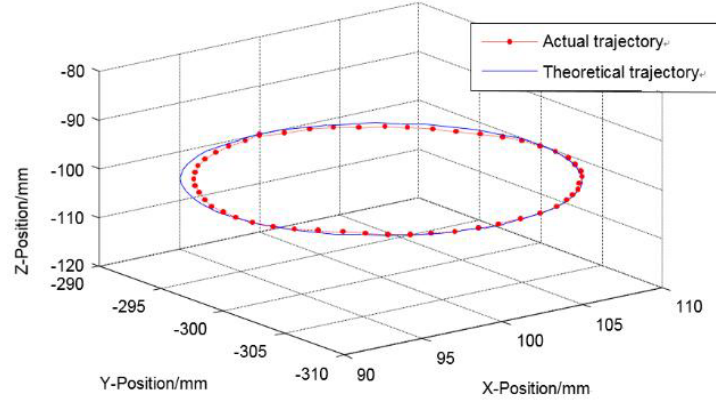

Fig. 6 shows the actual trajectory and theoretical trajectory of the WDPM moving platform which is tracking linear motion. Obviously, the actual and theoretical trajectory keep consistent all the time, but there are still some errors. The actual and theoretical trajectory curves of the WDPM moving platform are shown in Fig. 7, Fig. 8 and Fig. 9. According to the degree of curve fitting, the actual trajectory curves are consistent with the theoretical ones in the $\mathrm{X}, \mathrm{Y}$ and $\mathrm{Z}$ axis

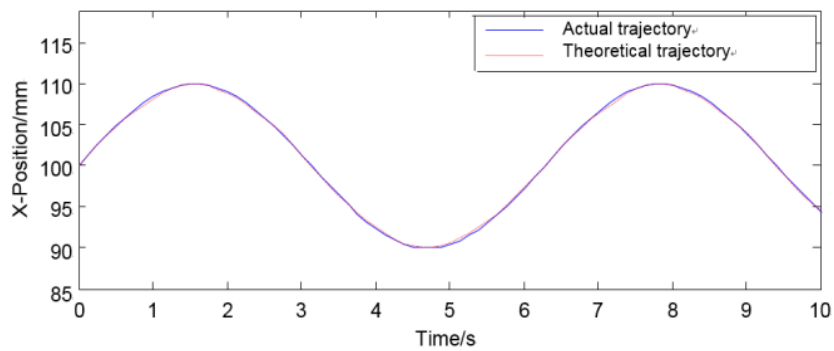

Fig. 7 Trajectories in $\mathrm{X}$ axis of the moving platform

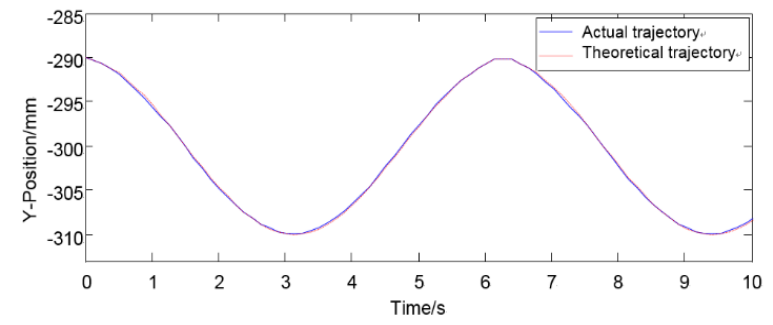

Fig. 8 Trajectories in $\mathrm{Y}$ axis of the moving platform

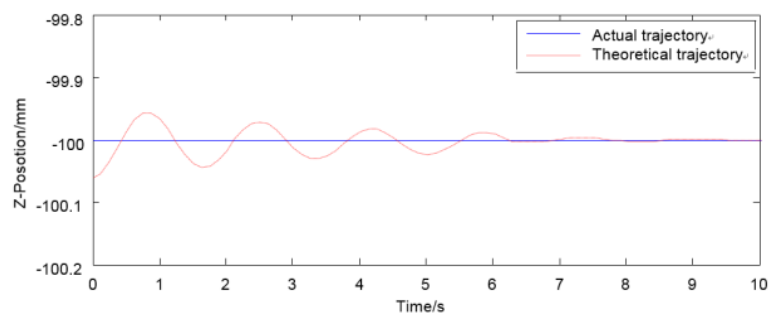

Fig. 9 Actual and theoretical trajectories in $\mathrm{Z}$ axis of the moving platform 


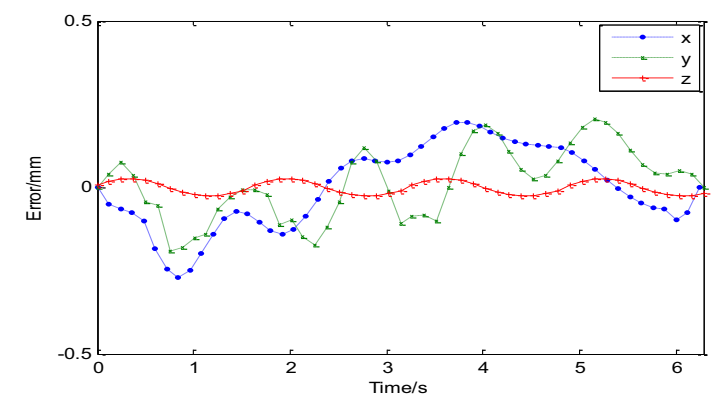

Fig. 10 Error curves of actual and theoretical trajectories

Fig. 10 shows the actual trajectory and theoretical trajectory of the WDPM moving platform which is tracking circular motion. Obviously, the actual and theoretical trajectory keep consistent and the error is reasonable.

The maximum error in $\mathrm{X}$ axis is $0.2836 \mathrm{~mm}$, the maximum error in $\mathrm{Y}$ axis is 0.2027 and the maximum error in $\mathrm{Z}$ axis is 0.0649 . Obviously, the error in $\mathrm{X}$ and $\mathrm{Y}$ axis is the largest and the error in $Z$ axis is the smallest. It shows that the error can be effectively suppressed in the control system.

Fig. 11 shows the tracking velocity curves of WDPM moving platform, the velocity of the moving platform is lower than $20 \mathrm{~mm} / \mathrm{s}$, comply with design requirements. Although the dynamic model and the control model of the WDPM system avoid the uncertainty of many parameters, errors and disturbances, in fact, there are no such systems without external interference. As a matter of fact, the co-simulation method introduced in this paper is still important for system analysis.

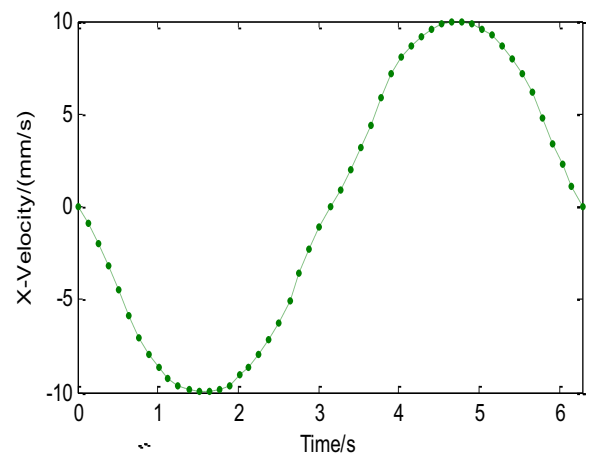

(a) Velocity in $\mathrm{X}$ axis

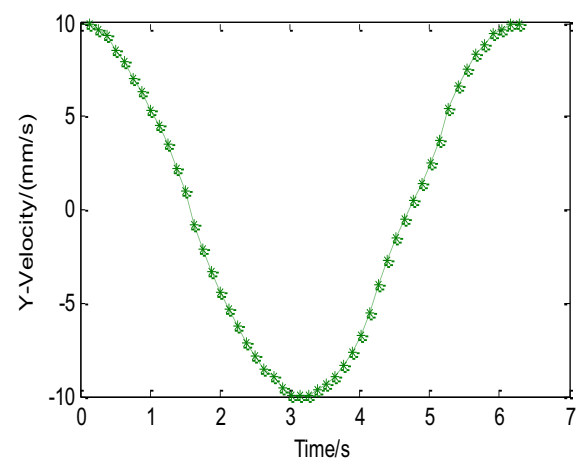

(b) Velocity in Y axis

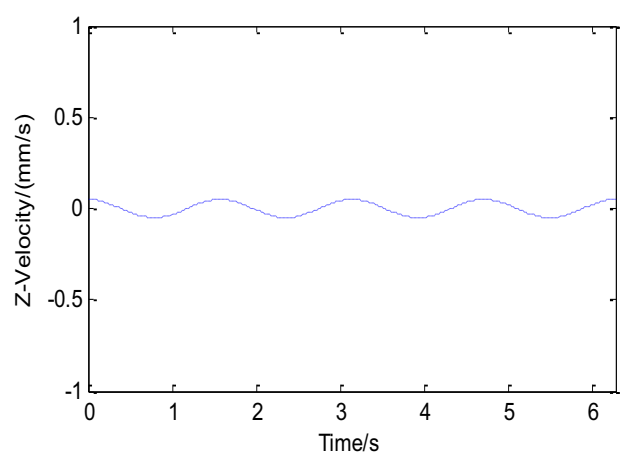

(c) Velocity in $Z$ axis

Fig. 11 Tracking velocity in three axis

\section{Conclusion}

Virtual prototype technology is used in dynamic simulation and control design of the WDPM system. The dynamic model of the 6-DOF WDPM is established by Lagrange method, the PD control method is used in the outer ring. According to the control system, MATLAB model is created and export dynamic model for tracking the moving platform of the WDPM system, and the cosimulation model is achieved. By analyzing the simulation results, verified the control effect of the control model and the results show that the designed control system can achieve the expected control effect.

\section{Acknowledgements}

This work was financially supported by the National Natural Science Foundation of China (51105303) and China Postdoctoral Science Foundation funded project (20110491673).

\section{REFERENCES}

1. Zi B , Zhu Z C , Du J L. Analysis and control of the cable-susporting system including actuator dynamics. Control Engineering Practice, 19(5):491-501, (2011)

1. LI Bing-cheng, YANG Dan, GU jin-feng. Kinematics simulation of 6-DOF parallel kinematic machine based on MATLAB and ADAMS. Modular Machine Tool,Automatic Manufacturing Technique, (11):28-31, (2009)

2. Aofei Tang, Yan Li, Hao Qua, Junbao Xiao. Dynamics modeling and simulating analysis of a Wire Driven Parallel Mechanism. Procedia Engineering, 15 (2011):788 - 794, (2011)

3. Diao X , Ma Ou. A method of verifying forceclosure condition for general cable manipulators with seven cables. Mechanism and Machine Theory, 42:1563-76, (2007)

4. Ablow C M, Schechter S. Numerical simulation of under sea cable dynamics. Ocean Engineering, 10(6): 443-57, (1983) 
5. Milinazzo F, Wilkie M, Latchman SA. An efficient algorithm for simulating the dynamics of towed cable systems. Ocean Engineering, 14(6): 513-26, (1987)

6. Huang S. Dynamic analysis of three-dimensional marine cables. Ocean Engineering, 21(6): 587-605, (1994)

7. Meunier G, Boulet B, Nahon M. Control of an overactuated cable-driven parallel mechanism for a radio telescope application. IEEE transactions on control systems technology, 17(5): 1043-54, (2009)

8. Kamman JW , Huston RL. Modeling of variable length towed and tethered cable systems. Journal of Guidance, Control and Dynamics, 22(4): 602-8, (1999)

9. Tang AF, Li Y, Qiu YY. Inverse dynamics of a long-span Wire Driven Parallel Robot. Mechanical science and technology for aerospace engineering, 29(4):435-40, (2010) (In Chinese) 